\title{
Effect of Bandwidth and Antenna Directivity on the Range Estimation Accuracy in a Multipath Environment
}

\author{
Zunnoor Tarique, Wasim Q. Malik, and David J. Edwards \\ Communications Research Group, Department of Engineering Science, \\ University of Oxford, Parks Road, \\ Oxford OX1 3PJ, UK \\ \{zunnoor.tarique, wasim.malik, david.edwards\}@eng.ox.ac.uk
}

\begin{abstract}
The accurate detection of the direct path in a dense multipath environment is vital for reliable performance of timebased and angle-of-arrival (AOA) based location estimation techniques. This paper investigates the effect of measurement bandwidth and antenna directivity on the detection of the direct path in a typical indoor scattering environment. It is experimentally shown that the ranging error diminishes with increase in measurement bandwidth and the standard deviation of the root mean square ranging error (RMSRE) is reduced by using directional transmit antennas. The results are obtained in a line-of-sight (LOS) propagation environment and are based on the time-of-arrival (TOA) based ranging technique.
\end{abstract}

Keywords- Multipath propagation; delay estimation; distance measurement; wideband propagation

\section{INTRODUCTION}

In the last decade, extensive research has been carried out on location and range estimation techniques. The work was triggered by the U.S. Federal Communications Commission passing a regulation, requiring wireless service providers to report the emergency callers' location to the public safety answering point within $50 \mathrm{~m}$ for mobile-based solutions and $100 \mathrm{~m}$ for network-based solutions, in $67 \%$ of the cases [1]. In 2003, the European Commission (EC) also passed a recommendation requiring the service providers to provide $\mathrm{E}$ 112 callers' location to emergency centers [2].

The location estimation techniques can be divided into three broad categories: received signal strength (RSS), angleof-arrival (AOA), and time. The RSS strength based techniques use the path loss models to calculate the distance between the transmitter and receiver from the received signal energy. The AOA based techniques rely on the angle of the incoming signal to determine the bearing between transmitter and receiver. The time based approaches, time-of-arrival (TOA) and time-difference-of-arrival (TDOA), measure the propagation delay between the transmitter and receiver to estimate the distance.

Among these categories, the accuracy of AOA, TOA, and TDOA suffer greatly in a multipath propagation, which is typical of a cluttered indoor environment where a large number

Z. Tarique acknowledges the financial support provided by the Rhodes Trust

W. Q. Malik's research is supported by EPSRC grant GR/T21769/01

of multipaths are received due to dense scattering [3]. In the line-of-sight (LOS) scenarios, the major source of error is the detection of the direct path from the delayed and overlapping replicas of the transmitted signal and noise. The accuracy can be improved by using wideband or ultrawideband (UWB) signals, as they provide higher time resolution $[4,5]$.

Besides the requirement for finer multipath resolution, there are two further types of errors associated with the multipath propagation, which also require minimization. Firstly, the early false alarm error is the detection of noise as a multipath before the arrival of the direct path. Secondly, the missed path error, which occurs if a multipath arriving after the direct path is falsely detected as the first path [6].

The high range estimation accuracy provided by wideband and UWB signals can be used in numerous applications, including tracking packages in warehouses, monitoring patients in hospitals, positioning rescue workers and fire fighters during natural disasters, and various military applications [4].

In this paper the effect of bandwidth and antenna directivity is experimentally investigated in a cluttered, indoor, LOS propagation environment. Rest of this paper is organized as follows. A brief overview of the TOA based range estimation is given in section II. The experimental setup is described in section III. Section IV presents the analysis and results regarding the effect of bandwidth and antenna directivity on range estimation accuracy in a wideband multipath environment. Concluding remarks are given in section $\mathrm{V}$.

\section{TOA BASED RANGE ESTIMATION}

The TOA based range estimation techniques rely on the accurate detection of the direct path and the determination of the associated propagation time, $\tau$, between the transmitter and receiver. The distance between the transmitter and receiver, $d$, is given by:

$$
d=c \tau=\sqrt{\left(x_{t}-x_{r}\right)^{2}+\left(y_{t}-y_{r}\right)^{2}}
$$

where $c$ is the speed of light, and $\left(x_{t}, y_{t}\right)$ and $\left(x_{r}, y_{r}\right)$ are the transmitter and receiver coordinates respectively. Equation (1) 
is the equation of a circle centered at the transmitter $\left(x_{t}, y_{t}\right)$. It is obvious that to locate a wireless device at least three transmitters are required, surrounding the device. The location of the wireless device is at the intersection of the three circles, as shown in Fig. 1.

\section{MEASUREMENT SETUP}

A Vector-Network Analyzer (VNA) based wideband channel sounder [7] was used for measurements over a bandwidth of $5 \mathrm{GHz}$, centered on $12.5 \mathrm{GHz}(10$ to $15 \mathrm{GHz}$ ), with 1601 frequency points. The transmit antenna was fixed, while the receive antenna was mounted on an X-Y positioner scanning an area of $0.75 \times 0.75 \mathrm{~m}^{2}$, with $0.015 \mathrm{~m}$ increments. The measured channel transfer function, $H(f)$, can be represented as:

$$
H(f)=\sum_{i=1}^{1601} a_{i} \delta\left(f_{s t}-i \Delta f\right) e^{j \phi_{i}}+n
$$

where $a_{i}$ is the amplitude of the ith frequency point, $\Delta f$ is the frequency step, $f_{s t}$ is the start frequency, $\varphi_{i}$ is the phase associated with the ith frequency point, and $n$ is the background noise. The measurements were calibrated to remove any phase error due to the measurement system. The antennas were not part of the calibration and the resulting signal distortion was considered part of the channel transfer function [8].

The measurements were conducted in a $6 \mathrm{~m} \times 6 \mathrm{~m}$ room, with concrete walls on three sides and glass window on the fourth. The floor layout of the room is shown in Fig. 2. The furniture, filling cabinets, and computers in the room not only depicted a typical office setting, but also provided a rich scattering environment. This allowed the range estimation analysis to be carried out in a dense multipath environment.

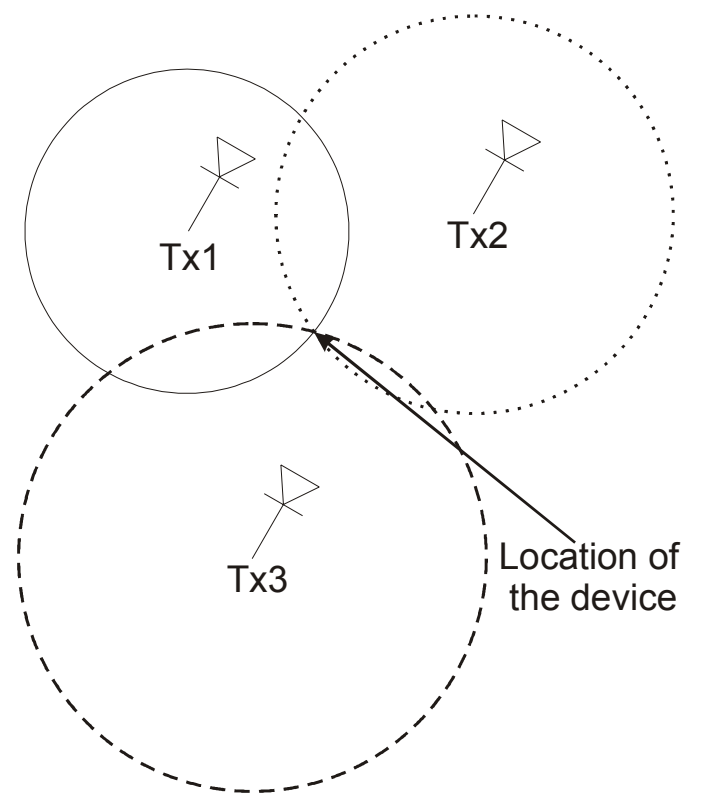

Figure 1. Locationing based on range estimation from three transmitters
The transmit and receive antennas were mounted $1.8 \mathrm{~m}$ above the floor. The transmit antennas were placed at three different locations around the $\mathrm{X}-\mathrm{Y}$ positioner, as shown in Fig. 2.

Discone omni-directional antennas [9] were used in the measurements. To analyze the effect of antenna directivity, the measurements were repeated with directional square horn antennas having approximately $20^{\circ}$ of half-power beam-width at $10 \mathrm{GHz}$ [10], at the transmitter.

\section{ANALYSIS AND RESULTS}

The measured channel transfer functions were windowed and converted to complex channel impulse response (CIR), $h(t)$, by applying inverse fast Fourier transform (IFFT). The CIR consists of direct path, reflected paths, and background noise. The CIR can be represented as:

$$
h(t)=\sum_{k=1}^{L} \alpha_{k} \delta\left(t-\tau_{k}\right)+n
$$

where $\alpha_{k}$ is the gain of the kth multipath, $\tau_{k}$ is the propagation delay of the kth multipath, $L$ is the number of multipaths, and $n$ is the background noise.

To reduce the early false alarm errors, a threshold was applied to suppress the noise and paths that are more than 10 $\mathrm{dB}$ below the dominant path. To determine the propagation time of the LOS path, a peak detection algorithm was applied to the thresholded CIR. The first detected peak was considered to be the LOS path.

\section{A. Effect of Bandwidth}

To analyze the effect of bandwidth on the range estimation accuracy for omni-directional antennas, the channel transfer function was divided into 5 frequency bands of $1,2,3,4$, and 5

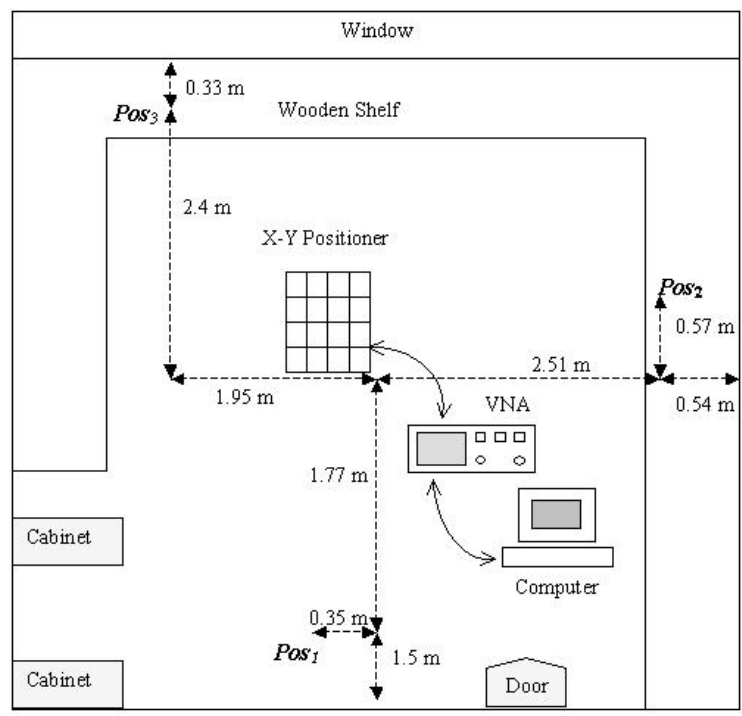

Figure 2. Floor layout of the room 
$\mathrm{GHz}$ respectively, centered on $12.5 \mathrm{GHz}$.

The root mean square ranging error (RMSRE) is the efficiency metric in the analysis and is calculated at each spatial point in the X-Y positioner. Table 1 shows the RMSRE for $50 \%, 70 \%$, and $90 \%$ accuracy levels, at various bandwidths. The results show that, as expected, the error decreases with increasing bandwidth. However, the decrease in the ranging error is highest when increasing the bandwidth from $1 \mathrm{GHz}$ to $2 \mathrm{GHz}$. This decrease in the ranging error is called "bandwidth gain" [11], and it reduces with further increase in the measurement bandwidth.

TABLE I. MSRE AT 50\%, 70\%, AND 90\% ACCURACY LEVELS FOR VARIOUS BANDWIDTHS USING OMNI-DIRECTIONAL ANTENNAS

\begin{tabular}{|l|l|l|l|}
\hline \multirow{2}{*}{$\begin{array}{c}\text { Bandwidth, } \\
\text { GHz }\end{array}$} & \multicolumn{3}{|c|}{ RMSRE, m } \\
\cline { 2 - 4 } & $\mathbf{5 0 \%}$ & \multicolumn{1}{|c|}{$\mathbf{7 0 \%}$} & $\mathbf{9 0 \%}$ \\
\hline 1 & 0.163 & 0.194 & 0.291 \\
\hline 2 & 0.084 & 0.1 & 0.136 \\
\hline 3 & 0.064 & 0.074 & 0.092 \\
\hline 4 & 0.053 & 0.062 & 0.076 \\
\hline 5 & 0.047 & 0.056 & 0.069 \\
\hline
\end{tabular}

The propagation time of each multipath is a function of the transmit and receive antennas' coordinates and the location of scatterers around them. An increase in the bandwidth improves the timing resolution of the measurement system. However, for optimal range estimation accuracy, the measurement system only has to distinguish between the direct path and the second arriving multipath. Therefore, if the measurement bandwidth is large enough to identify the first arriving multipath in the LOS system, then further increase in the bandwidth will not offer higher gain in range resolution. So an infinitely large bandwidth for accurate range estimation is not necessary and a bandwidth of $4 \mathrm{GHz}$, at $12.5 \mathrm{GHz}$, is sufficient for reliable range estimation [11]. Therefore, for the analysis of antenna directivity on range estimation accuracy, only the bandwidth of $5 \mathrm{GHz}$ is considered.

\section{B. Effect of Antenna Directivity}

To investigate the effect of antenna directivity, the experiment and analysis were repeated with directional squarehorn transmit antennas, while keeping the receive antenna omni-directional, so that multipaths arriving from all directions were received. A threshold of $30 \mathrm{~dB}$ was applied to suppress the noise and reflected paths.

Fig. 3 compares the cumulative distribution function (CDF) of the spatial variation of RMSRE for omni-directional and directional transmit antennas over $5 \mathrm{GHz}$ bandwidth, with mean taken over three transmitters at a particular spatial point. For clarity, Table II shows the RMSRE for $50 \%, 70 \%$, and $90 \%$ accuracy levels. The omni-directional antennas exhibit greater variance in RMSRE than the directional antennas. Secondly, the CDF curve corresponding to the omni-directional antennas has longer tail than the curve for the directional one. It is also shown in Table III, that omni-directional transmit

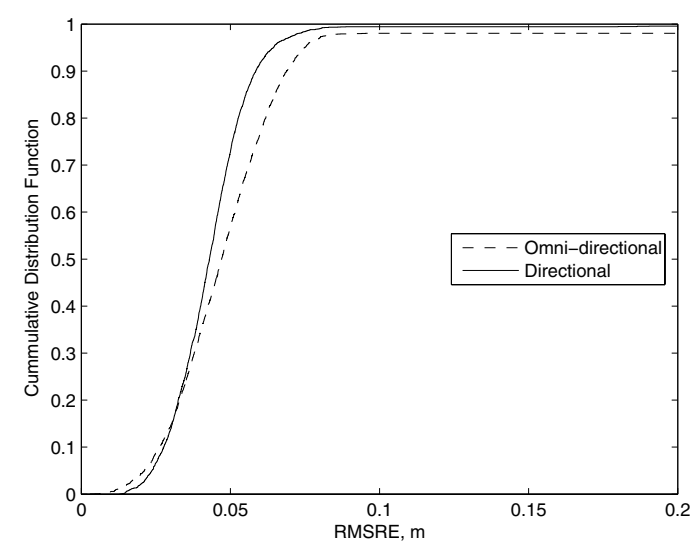

Figure 3. CDF of the spatial variation of RMSRE for omni-directional and directional transmit antennas operating over $5 \mathrm{GHz}$ bandwidth

TABLE II. RMSRE AT 50\%, 70\%, AND 90\% ACCURACY LEVELS FOR 5 GHz BANDWIDTH USING DIRECTIONAL TRANSMIT ANTENNAS

\begin{tabular}{|l|l|}
\hline $\begin{array}{c}\text { Accuracy } \\
\text { Level }\end{array}$ & RMSRE, m \\
\hline $50 \%$ & 0.043 \\
\hline $70 \%$ & 0.049 \\
\hline $90 \%$ & 0.058 \\
\hline
\end{tabular}

antennas exhibit 15 times higher standard deviation in the RMSRE than directional transmit antennas. Furthermore, the maximum RMSRE is greater for omni-directional transmit antennas, which explains the long tail of the CDF in Fig. 3.

TABLE III. COMPARISON OF RMSRE PARAMETERS OF OMNIDiRECTIONAL AND DiRECTIONAL TRANSMIT ANTENNAS

\begin{tabular}{|l|l|l|}
\hline \multicolumn{1}{|c|}{ Parameters } & \multicolumn{1}{|c|}{$\begin{array}{c}\text { Omni-directional } \\
\text { Transmit } \\
\text { Antennas }\end{array}$} & \multicolumn{1}{c|}{$\begin{array}{c}\text { Directional } \\
\text { Transmit } \\
\text { Antennas }\end{array}$} \\
\hline Std. Dev ${ }^{*} \mathrm{~m}$ & 0.159 & 0.019 \\
\hline Maximum, m & 1.698 & 0.283 \\
\hline
\end{tabular}

From the results presented in this subsection, it is clear that the major advantage of using directional transmit antennas instead of omni-directional transmit antennas is the reduction in the error variance. This decrease in standard deviation is, firstly, due to antenna directivity, as transmitting the power in only one direction in the indoor channel reduces the number of effective scatterers and the angular spread, which are otherwise substantial. This increases the Ricean $\mathrm{K}$ factor, ratio of the deterministic signal power to the multipath variance [12]. This causes an increase in the probability of direct path being the dominant path [13], hence reducing the missed path errors. Secondly, the delay between the first and second multipath is greater for directional antennas than omni-directional antennas, since some of the paths in between no longer exist for 
directional antennas. This facilitates the detection of the direct path.

\section{CONCLUSION}

Missed path errors and early false alarm errors depend on the antenna surroundings and distance between transmitter and receiver. The range estimation accuracy increases with measurement bandwidth but the decrease in RMSRE diminishes with measurement bandwidth. This is because increasing the bandwidth further than required to distinguish between consecutive multipaths does not yield any gain and will result in the wastage of resource. Secondly, the standard deviation of ranging errors can decrease about 15 times by using directional transmit antennas. This results from the reduction of number of received multipaths and increase in the probability of direct path being the dominant path as well. These results have been obtained for LOS propagation but are extendable to NLOS propagation scenarios.

\section{REFERENCES}

[1] "Revision of the commission's rules to insure compatibility with enhanced 911 emergency calling systems, RM-8143," U.S. Federal Communications Commission, FCC Docket No. 94 - 102, Jul. 1996.

[2] "Commission pushes for deployment of location enhanced 112 emergency services," EU Institutions Press Release, Brussels DN: IP/03/1122, 25 Jul. 2003.
[3] H. Hashemi, "The indoor radio propagation channel," Proc. IEEE, vol. 81, no. 7, pp. 943 - 968, Jul. 1993.

[4] S. Gezici, et al., "Localization via ultra-wideband radios: a look at positioning aspects of future sensor networks," in IEEE Sig. Proc. Mag., vol. 22, 2005, pp. $70-84$.

[5] W. Q. Malik, D. J. Edwards, and C. J. Stevens, "Optimal System design considerations for ultra-wideband multipath channel," in Proc. IEEE Veh. Tech. Conf., VTC-2005, Fall. Dallas, TX, USA, 2005.

[6] J-Y. Lee and R. A. Scholtz, "Ranging in a dense multipath environment using an UWB radio link," IEEE J. Sel. Areas Commun., vol. 20, no. 9, pp. 1677 - 1683, Nov. 2002.

[7] A. M. Street, L. Lukama, and D. J. Edwards, "Use of VNAs for wideband propagation measurements," IEE Proc. - Commun., vol. 148, no. 6 , pp. 411 - 415, Dec. 2001.

[8] W. Q. Malik, D. J. Edwards, and C. J. Stevens, "Angular-spectral antenna effects in ultra-wideband communication links," IEE Proc. Commun. (in press).

[9] R. W. P. King, Theory of Linear Antennas. Cambridge, MA, USA: Harvard Press, 1956.

[10] J. D. Kraus, Antennas, Second ed: McGraw-Hill International Editions, 1988.

[11] Z. Tarique, W. Q. Malik, and D. J. Edwards, "Bandwidth requirements for accurate detection of direct path in multipath environment," Elec. Lett., vol. 42, no. 2, pp. 100 - 102, Jan. 2006.

[12] T. S. Rappaport, Wireless Communications: Principles \& Practice: Prentice Hall, 1996.

[13] J. A. Dabin, N. Ni, A. M. Haimovich, E. Niver, and H. Grebel, "The effects of antenna directivity on path loss and multipath propagation in UWB indoor wireless channels," in IEEE UWBST. Reston, VA, USA, 2003. 